\title{
Interactive JIMD articles using the iSee concept: turning a new page on structural biology data
}

\author{
Wen Hwa Lee - Wyatt W. Yue • Eugene Raush • \\ Maxim Totrov • Ruben Abagyan • Udo Oppermann • \\ Brian D. Marsden
}

Received: 22 March 2011 /Revised: 22 March 2011 / Accepted: 4 April 2011 /Published online: 21 April 2011

(C) SSIEM and Springer 2011

Since its inception, researchers in structural biology have reported detailed molecular models of macromolecules involved in the functioning of the human body. This wealth of information has provided a better understanding of the molecular mechanisms by which the proteins and enzymes perform their biochemical and physiological functions. Several scientific fields have benefited from these advance-

Communicated by: Verena Peters

Competing interest: None declared.

W. H. Lee · W. W. Yue · U. Oppermann · B. D. Marsden

Structural Genomics Consortium, University of Oxford,

Oxford OX3 7DQ, UK

E. Raush $\cdot$ M. Totrov $\cdot$ R. Abagyan

Molsoft LLC,

San Diego, CA 92121, USA

U. Oppermann

Botnar Research Centre,

NIHR Oxford Biomedical Research Unit,

Oxford OX3 7LD, UK

B. D. Marsden

Nuffield Department of Clinical Medicine, University of Oxford, Oxford OX3 7BN, UK

W. H. Lee $(\varangle) \cdot$ B. D. Marsden $(\varangle)$

SGC, ORCRB Old Road Campus, University of Oxford,

Roosevelt Drive,

Oxford OX3 7DQ, UK

e-mail: wenhwa.lee@sgc.ox.ac.uk

B. D. Marsden

e-mail: brian.marsden@sgc.ox.ac.uk ments, including clinicians and those studying inherited metabolic disorders caused by mutational defects in proteins. Furnished with structural data, scientists are able to make sense of the different mutations observed in patients and understand their effects at the protein level (Yue and Oppermann 2011).

In recent years, advances in high-throughput methods and concerted structural genomics efforts have yielded a large increase in the number of publically available solved structures. The Structural Genomics Consortium (SGC) was created in 2004 to tackle the challenging task of solving structures of medically relevant human proteins in a high-throughput manner (Gileadi et al. 2007; Weigelt 2010). While contributing to over $20 \%$ of novel protein structures in the public domain, the SGC recognised that dissemination of its results and data beyond the boundaries of structural biology is fundamental in promoting tangible advancements in human health and medicine. This has been the main motivation behind the development of the iSee concept by the SGC, in collaboration with MolSoft L.L.C., as a means to enable flexible, instructive and intuitive data dissemination (Abagyan et al. 2006).

The iSee concept provides a flexible and intuitive tool to deliver text annotation and easy-to-use interactive molecular graphics to authors and readers alike. In one single file, the authors can include many different data types, including full atomic coordinates of protein structures or chemical compounds, DNA and amino acid sequences, images, tables, text and molecular graphics animations. These files can be created, viewed and edited using the free ICM Browser software provided by 


\section{iSee 6 First Time Plugin Installation (to read enhanced article online)}

\section{Support: isee@sgc.ox.ac.uk}

- Identify and click on the link in the JIMD article. This will take you to the SSIEM's website with the enhanced iSee version of the article.

- You will reach a page which looks like this:

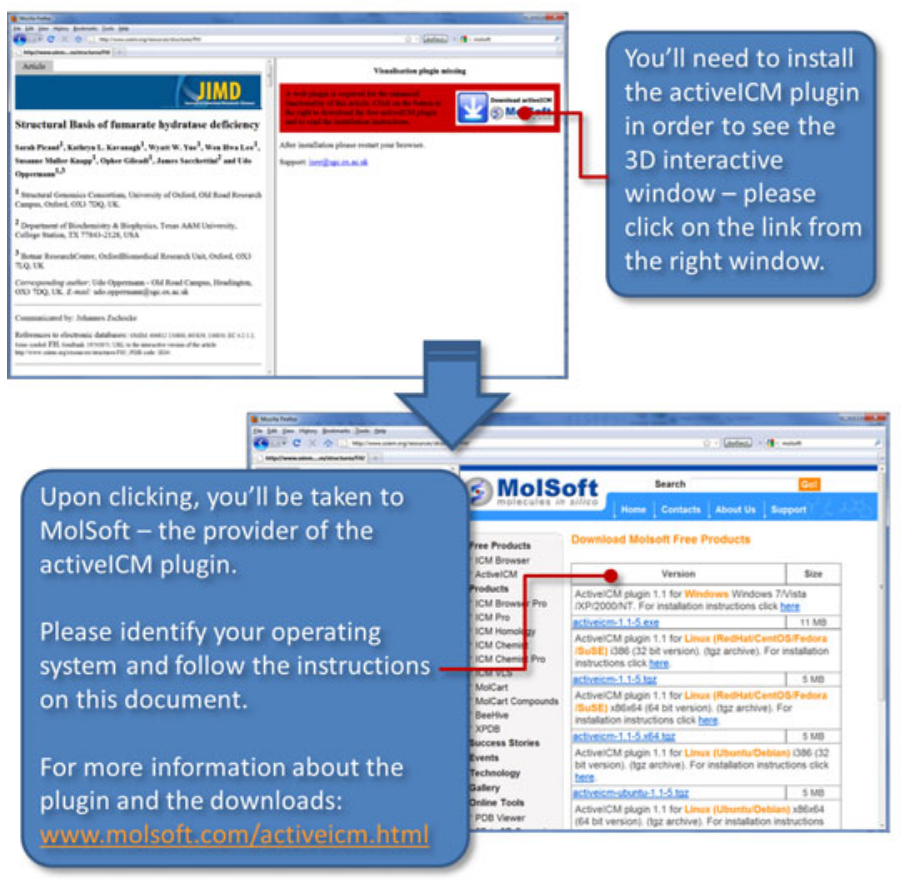

Windows (XP, Vista, Windows 7)

- Download and run the latest installer and follow the installation instructions

- Close all web browser windows and restart web browser

- $\quad$ Supported web browsers: Firefox, IE, Opera or Chrome

\section{Mac OS X}

- Download the latest version

Safari: the installer will be launched automatically Firefox: please refer to Molsoft instructions

- Close all web browser windows and restart web browser

- Supported web browsers: Safari 32bit, Firefox

- Safari 64bit users: Please refer to Molsoft instructions

\section{Linux}

- Installation instruction for Linux is dependent on the distribution that you are using. Please refer to Molsoft instructions for your case.

- Close all web browser windows and restart web browser

- Supported web browser: Firefox

After restarting your browser, open again the link to the enhanced version of the article.

Fig. 1 Instructions for the installation of the activeICM web plugin that will enable the molecular graphics functions on the JIMD interactive articles

MolSoft, available for Windows, Mac and Linux platforms (Raush et al. 2009). A web browser plugin has also been developed which allows the iSee concept to be used directly on the web (Raush et al. 2009). This has opened the doors for anyone wishing to share their structural findings - either through their own websites (http://www.thesgc.org/iSee) or as peer-reviewed articles published in scientific journals such as PLoS ONE, PLoS Biology and Nature Structure \& Molecular Biology (Lee et al. 2009; Li et al. 2011; Morris et al. 2010; Rellos et al. 2010).

We are therefore excited to work with the Society for the Study of Inborn Errors of Metabolism (SSIEM) and the Journal of Inherited Metabolic Disease (JIMD) to bring the iSee concept to all involved in the study of metabolic enzymes, by introducing the first interactive article in this issue of JIMD. The article provides a 3D structure-mutation mapping of the protein fumarate hydratase and allows the reader to investigate the causal relationship between protein sequence, structure and disease phenotypes. We hope that the interactive articles will help us, structural biologists, to communicate our data and exchange ideas with the clinical community in a more approachable fashion, closing the gap between research communities and promoting further collaborations.

Instructions on how to install and use the web plugin are provided in Figs. 1 and 2.

Acknowledgements The Structural Genomics Consortium is a registered charity (Number 1097737) funded by the Canadian Institutes for Health Research, the Canadian Foundation for Innovation, Genome Canada through the Ontario Genomics Institute, GlaxoSmithKline, KarolinskaInstitutet, the Knut and Alice Wallenberg Foundation, the Ontario Innovation Trust, the Ontario Ministry for Research and Innovation, Merck and Co., Inc., the Novartis Research Foundation, the Swedish Agency for Innovation Systems, 


\section{iSeelo iSee - Quick Reference Chart}

There are two main windows in every iSee session - one with text and static images (left) and another which allows interaction with 3D objects (right). The features and behaviours for each window are described below. Support: isee@sgc.ox.ac.uk

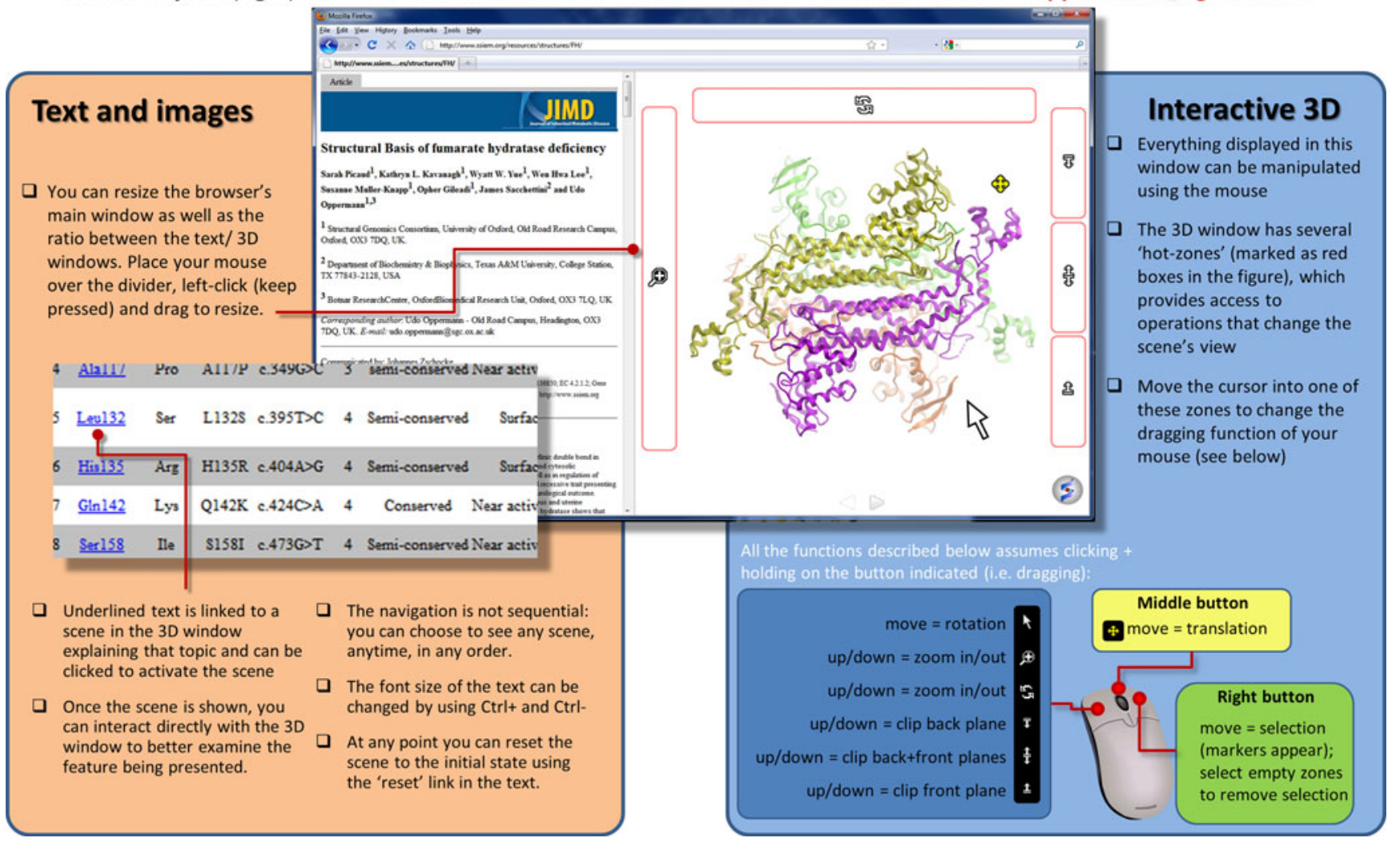

Fig. 2 Instructions on how to use the molecular graphics features of the activeICM plugin

the Swedish Foundation for Strategic Research and the Wellcome Trust. This work is also supported by the NIHR Oxford Biomedical Research Unit. ICM browser and its derivative, activeICM, used in the JIMD iSee enhanced articles have been developed at Molsoft, LLC.

\section{References}

Abagyan R, Lee WH, Raush E et al (2006) Disseminating structural genomics data to the public: from a data dump to an animated story. Trends Biochem Sci 31(2):76-78

Gileadi O, Knapp S, Lee WH et al (2007) The scientific impact of the Structural Genomics Consortium: a protein family and ligand-centered approach to medically-relevant human proteins. J Struct Funct Genomics 8(2-3):107-119

Lee WH, Atienza-Herrero J, Abagyan R et al (2009) SGCstructural biology and human health: a new approach to publishing structural biology results. PLoS ONE 4(10): e7675

Li M, Dimaio F, Zhou D et al (2011) Crystal structure of XMRV protease differs from the structures of other retropepsins. Nat Struct Mol Biol 18(2):227-229

Morris JH, Meng EC, Ferrin TE (2010) Computational tools for the interactive exploration of proteomic and structural data. Mol Cell Proteomics 9(8):1703-1715

Raush E, Totrov M, Marsden BD et al (2009) A new method for publishing three-dimensional content. PLoS ONE 4(10):e7394

Rellos P, Pike AC, Niesen FH et al (2010) Structure of the CaMKIIdelta/calmodulin complex reveals the molecular mechanism of CaMKII kinase activation. PLoS Biol 8(7):e1000426

Weigelt J (2010) Structural genomics-impact on biomedicine and drug discovery. Exp Cell Res 316(8):1332-1338

Yue WW, Oppermann U (2011) High-throughput structural biology of metabolic enzymes and its impact on human diseases. J Inherit Metab Dis 